\title{
Antibacterial Properties of Functionalized Gold Nanoparticles and Their Application in Oral Biology
}

\author{
Chen Su, ${ }^{1}$ Kun Huang, ${ }^{1}$ Hao-Hong Li, ${ }^{2}$ You-Guang Lu $\mathbb{D},{ }^{3}$ and Da-Li Zheng $\mathbb{D}^{4}$ \\ ${ }^{1}$ School of Stomatology, Fujian Medical University, Fuzhou 350122, China \\ ${ }^{2}$ College of Chemistry, Fuzhou University, Fuzhou, Fujian 350116, China \\ ${ }^{3}$ Department of Preventive Dentistry, School and Hospital of Stomatology, Fujian Medical University, Fuzhou 350001, China \\ ${ }^{4}$ Fujian Key Laboratory of Oral Diseases, Fujian Biological Materials Engineering and Technology Center of Stomatology, School and \\ Hospital of Stomatology, Fujian Medical University, Fuzhou 350004, China
}

Correspondence should be addressed to You-Guang Lu; fjlyg63@fjmu.edu.cn and Da-Li Zheng; dalizheng@fjmu.edu.cn

Received 29 June 2020; Revised 17 October 2020; Accepted 20 October 2020; Published 18 November 2020

Academic Editor: Angelo Taglietti

Copyright ( 92020 Chen Su et al. This is an open access article distributed under the Creative Commons Attribution License, which permits unrestricted use, distribution, and reproduction in any medium, provided the original work is properly cited.

\begin{abstract}
As bacterial resistance is becoming increasingly serious, the development of antibacterial nanomaterials is an effective method of solving this problem. Gold nanoparticles have good stability and excellent biocompatibility and are easily modified, and their antibacterial properties can be enhanced by changing their structure and size or adding ingredients. Gold nanoparticles are also excellent drug carriers that can improve the antibacterial effects of loaded antibacterial drugs. After being modified and combined with other antibacterial drugs, gold nanoparticles can also play a better antibacterial role for effective antibacterial strategies against some resistant bacteria. Gold nanoparticles have photothermal effects, and modified gold nanoparticles can be a good medium for photothermal treatments to kill bacteria. By adding functionally modified gold nanoparticles, many materials can obtain much needed antibacterial properties. Gold nanoparticles can also be combined with cations, lowtemperature plasma, various surface ligands, and other potential antibacterial agents. In short, the antibacterial characteristics of functionalized gold nanoparticles demonstrate that they have considerable practical application value and provide more ideas to solve antibacterial problems. At the same time, the application of gold nanoparticles in oral biology is also increasing.
\end{abstract}

\section{Introduction}

Because of the emergence and spread of drug resistance in bacterial pathogens, antibiotics often lose their effectiveness over time. The so-called "antibiotic resistance crisis" and iatrogenic infections caused by drug-resistant bacteria result in additional medical costs of up to billions of dollars annually [1-3]. Faced with this increasingly severe situation, discovering new antibacterial agents and therapeutic strategies is urgently necessary. Nanoparticles provide a universal platform for therapeutic applications based on their own unique physical and chemical properties and provide treatment for drug-resistant bacteria $[3,4]$. Antibacterial activity exhibited by nanomaterials such as silver, gold, copper, titanium, zinc oxide, and magnesium oxide is expected to become a substitute for antibacterial agents [5]. The unique properties of gold nanomaterials, such as their adjustable size, shape, surface properties, optical properties, biocompatibility, low cytotoxicity, high stability, and multi-functional potential, make them appealing in many fields of medicine [6]. According to its nanostructure, high surface volume characteristics, and biocompatibility, some scholars have already applied gold nanoparticles (GNP) to experiments on gum disease, dental caries, tissue engineering, dental implantology, and cancer diagnosis. GNP has antifungal and bacterial activity, so it can be incorporated into certain biological materials to impart antibacterial properties to the material, thereby improving the application value of the material [7]. Antibacterial drugs can bind to nanoparticles via noncovalent or covalent bonds using gold nanoparticles as carriers for antibacterial drugs, so that antibacterial drugs reach the site of action more effectively, improving their antibacterial effects. Gold nanoparticles have photothermal effects that can play sterilizing roles under continuous laser irradiation $[2,4,8]$. 
Gold is considered a nontoxic nanomaterial, but the substances used for preparation and modification may be toxic. This toxicity may be manifested when the concentration of gold nanoparticles is high, but gold nanoparticles produce obvious antibacterial effects. At certain concentrations, they did not show toxic effects on normal cells $[2,4,9,10]$. Modified gold nanoparticles not only demonstrate good antibacterial activity against standard strains but also have unique antibacterial activity against multidrug-resistant bacteria. After multiple generations of cultivation, it is not easy to induce bacteria that are resistant to gold nanoparticles [11]. Nowadays, with the rapid development of nanoscience and nanotechnology, it has proposed solutions different from traditional methods for people to diagnose, resist, and overcome various diseases. At present, metal nanoparticles have been developed, mainly silver and gold nanoparticles (AgNPs and AuNPs, respectively), which can be applied to many aspects in the medical and pharmaceutical fields, including antibacterial, antibiofilm, drug delivery systems, diagnostic tools, and Personal care products and cosmetics [12]. This article primarily summarizes the recent research progress into functionalized gold nanoparticles in mixed antibacterial and the application of gold nanoparticles in oral biology. We briefly explain synthesis methods, antibacterial effects, antibacterial mechanisms, applications in oral biology, and shortcomings to provide a reference for future research.

\section{Gold Nanoparticle Synthesis Methods}

In recent years, whether in the fields of biomedicine, food, or electronics, the use of nanotechnology and nanomaterials has increased. Gold nanoparticles (AuNPs) are one of the important branches of nanometal research. There are currently more than 1,000 methods available for synthesizing gold nanoparticles $[13,14]$. The formation of nanoparticles by decomposition of high-energy radiation or electron bombardment based on transmission electron microscopy is widely known. There are various methods of synthesizing gold nanoparticles, including chemical synthesis, physical synthesis, and biosynthesis. Innovative and environmentally friendly nanotechnology-based methods have recently emerged mainly using biological resources to produce nanostructures with unique antibacterial properties. To this end, Rónavári et al. biosynthesized gold nanoparticles using cellfree extract of erythrocyte yeast $R$. cerevisiae [15]. For an eco-friendly approach, natural plant extracts contain a variety of metabolites, including carbohydrates, alkaloids, terpenes, phenolic compounds, and enzymes. Biomolecules in plant extracts can reduce metal ions to nanoparticles through a one-step and environmentally friendly synthesis process [16]. Fierascu et al. described the plant-mediated synthesis of metal nanostructures (gold and silver) using an ethanol extract of Melissa officinalis L. (obtained by accelerated solvent extraction) [17]. In the study, Doan et al. used the water extract of waste corncobs to prepare cost-effective and environmentally friendly silver and gold nanoparticles. The formation of metal nanoparticles (MNP) is optimized by the UV-Vis method. Gold nanoparticles (CC-AuNPs) show high antibacterial activity against three bacterial strains including Salmonella typhimurium, Bacillus cereus, and Staphylococcus aureus [18]. Gold nanoshells also have interesting physical properties, including optical and surface plasmon resonance, which can be customized during the synthesis process, giving gold nanoshells significant potential in nanomedicine [19].

\section{Antibacterial Effects of Gold Nanomaterials}

3.1. Antibacterial Effects of Pure Gold Nanoparticles. Some scholars have proposed using trisodium citrate (TSC) as the first reducing agent and chlorogenic acid (CGA) as the second reducing agent to quickly and simply synthesize gold nanoparticles (CGA-AuNPs) with different particle sizes. Both CGA-AuNPs and CGA have excellent antibacterial activity. Even without adding other stabilizers, the synthesized CGA-AuNPs can still maintain good condition even after 26 days [20]. Murphin et al. achieved the green synthesis of dispersed cuboid gold nanoparticles using algae. An antibacterial test showed that the synthesized gold nanoparticles had certain potential for human pathogens, E. coli, and Staphylococcus aureus [21]. Ali et al. used the stems of the Ayurvedic medicinal plant Tinospora cordifolia to synthesize gold nanoparticles (AuNPs) and studied the effect of AuNPs on the biofilm of Pseudomonas aeruginosa PAO1. The results show that green synthetic AuNPs can be used as effective nanoantibiotics to combat biofilm-related infections caused by Pseudomonas aeruginosa [22]. Mocan et al. used light to stimulate gold nanoparticles synthesized by standard wet chemical procedures and then further applied them to MRSA bacteria to increase the MRSA necrosis rate in a short cultivation time [23]. Singh et al. used the aqueous extract of C. sativa stem to synthesize gold nanoparticles without any additional reducing, stabilizing and capping agents. The synthesized nanoparticles were crystalline with an average diameter between 12 and $18 \mathrm{~nm}$, and showed bactericidal effects against Pseudomonas aeruginosa and Escherichia coli. [24]. Arafa et al. reported in vitro and in vivo studies demonstrating that AuNP thermosensitive gels have certain bioavailability, skin permeability, and antibacterial and anti-inflammatory activities [25].

\subsection{Antibacterial Effects of Functionalized Gold}

Nanoparticles. Researchers' views on the antibacterial effects of pure gold nanoparticles are not uniform. Many scholars believe that pure nanogold particles have no antibacterial effects or the effects are not obvious. Zhang et al. found that gold nanoparticles generally do not have bactericidal effects or have only weak bactericidal effects at high concentrations. Gold nanoparticles may have bactericidal effects because the chemicals (gold ions, surface coating agents, and chemicals involved in synthesis) coexisting in gold nanoparticles are not completely removed [26]. Burygin et al. used several methods to study the effect of $15 \mathrm{~nm}$ gold nanoparticles on gentamicin's antibacterial activity. Within the range of experimental errors, no differences were found in the antibacterial activity of gentamicin and gentamicin-gold nanoparticle mixtures at various concentrations of gentamicin and particles. Gold nanoparticles that precipitated in the conjugate 
had no antibacterial activity, while the gentamicin-gold nanoparticle mixture and free gentamicin supernatant had the same activity [27]. Data from Payne et al. showed that citrate-gold nanoparticles had no antibacterial activity compared to kanamycin-gold nanoparticles. Regardless of the mechanism, gold nanoparticles seem to rely on binding to active antibiotics [28]. Brown et al. reported that pure gold nanoparticles had no antibacterial effect, but can be used as good antibiotic drug delivery systems. Gold nanoparticles have antibacterial properties only when ampicillin binds to their surfaces. Ampicillin-functionalized AuNP is an effective broad-spectrum fungicide that can resist Gram-negative and Gram-positive bacteria [29]. Alsamhary et al. used flavonoid glycerin as a reducing agent and capping agent for synthetic gold nanoparticles (AuNPs). In addition, the antibacterial efficacy of synthetic AuNP against opportunistic bacterial pathogens that cause respiratory infections was evaluated. Antibacterial studies confirmed the broad-spectrum antibacterial activity of AuNPs against the tested Gram-positive and Gram-negative bacteria [30]. Mohamady Hussein et al. reported a green synthesis method of gold-chitosan hybrid nanoparticles (Au-CS hNPs) using different concentrations of CS as a capping agent/reducing agent to study the physicochemical properties of the developed Au-CS hNPs by CS concentration and antibacterial activity. Its antibacterial activity is proportional to the amount of CS used in the preparation process. A large amount of CS is required to prepare highly stable Au-CS hNP with small size, uniform shape, and strong antibacterial/antifungal properties [31]. Therefore, the antibacterial effects of pure gold nanoparticles are not ideal but can be used as good active carriers. After modification, the antibacterial effects can be improved. Functionalized gold nanoparticles (GNPs) with controlled geometric and optical properties have become the subject of extensive research and biomedical applications. Gold nanoparticles can be used as drug carriers to provide or transport drugs in a targeted manner and can solve important problems, such as multidrug resistance. Multifunctional gold-based composite materials can also be used for combination therapy [32]. Govindaraju et al. reported a new method of synthesizing glucosamine-functionalized gold nanoparticles. Gold nanoparticles were exposed to ultraviolet and laser light during experiments. The results demonstrated that functionalized gold nanoparticles and ultraviolet and laser-irradiated gold nanoparticles had better antibacterial effects than pure gold nanoparticles [33]. Bajaj et al. observed that gold nanoparticles at the end of peptides had higher antibacterial and antifungal effects than gold nanoparticles at the end of peptides in natural peptides and unbound metal nanoparticles. They found that the killing effect of cationic peptide (1-His1-Arg-OMe)-capped gold nanoparticles was even better than known antibiotics [34]. Jiang et al. reported that gold nanoparticles do not have antibacterial activity, but by modifying their surface or preparing gold and platinum bimetallic nanoparticles with different composition ratios, the antibacterial spectrum and antibacterial properties of nanoparticles can be adjusted. When mercaptopyrimidine molecules that do not have antibacterial activity are modified on the surface of gold particles, the gold nanoparticles exhibit good antibac- terial activity against Gram-negative bacteria and multidrugresistant negative bacteria. It was also reported that a class of nonantibiotic drugs and gold nanoparticles coated with mercaptopyrimidine can produce a broad-spectrum antibacterial and bactericidal effect on super bacteria [8]. Wang et al. designed antibacterial peptide/triclosan comodified gold nanoparticles. They had better antibacterial effect than pure gold nanoparticles [35]. Tian et al. found that using small molecule analogs or unmodified gold nanoparticles alone did not produce antibacterial activity, and the modified gold nanoparticles only demonstrated good antibacterial activity against standard strains. Multidrug-resistant bacteria also have unique antibacterial activities. After multigeneration culture, it is not easy to induce bacteria that are resistant to gold nanoparticles [11].

3.2.1. Transport Medicines. Jabir et al. used modified glutathione gold nanoparticles as a drug delivery system for linalool. Linalool alone had low activity against Gram-positive and Gram-negative bacteria, but the experimental results showed that linalool gold nanoparticles (LIN-GNPs) were effective against Gram-positive bacteria. The functional mechanism of LIN-GNP acts on the bacterial cell membrane, resulting in the loss of integrity and increased cell wall permeability and stimulation of ROS production, causing bacterial nucleic acid damage [36]. Lin et al. combined mesoporous silica with excellent performance as a nanodrug carrier and a gold nano double cone with unique photothermal properties to construct an intelligent drug-loading system with two hybrid nanoparticles for antibacterial activity of periodontal pathogens. After stirring the antibacterial drug with the carrier for $24 \mathrm{~h}$, the drug-loading efficiency reached $76.5 \%$, confirming its excellent drug-loading capacity. Experiments showed that near-infrared light irradiation can significantly increase the cumulative release of drugs and has good near-infrared response. Antibacterial experiments further confirmed that using this carrier to carry drugs significantly inhibited the growth of bacteria, with an inhibition rate of 90\% [37]. Zhao and Jiang loaded small drug precursor molecules on gold nanoparticles. Experiments confirmed that the nanoparticles had good antibacterial effects on standard Gram-negative bacteria and multidrug-resistant clinical isolates. Compared with commercial antibiotics, this kind of nanogold rarely develops drug resistance. Further studies showed that gold nanoparticles disrupted the structure of bacterial cell membranes, causing cell contents to leak and leading to bacterial death. However, at high concentrations, gold nanoparticles have no significant toxicity to human primary cells, so the nano-antibacterial agent is expected to be used in clinical treatment [38]. Cao et al. used gold nanoparticles as a carrier for sound-sensitive drugs (ciprofloxacin) in SACT. They found that under low-concentration ciprofloxacin, extended ultrasound times, and increased temperatures, the gold nanoparticles significantly enhanced the bacteriostatic effect of ciprofloxacin and ultrasound on E. coli, inferring that AuNPs have a certain promotion effect on the bacteriostatic effects of SACT [39]. Kalita et al. reported an efficient antibacterial hybrid prepared by surface functionalization of lysozyme-capped gold nanoclusters with $\beta$-lactam antibiotic 
ampicillin. The prepared hybrid not only made MRSA resistant to ampicillin but also showed enhanced antibacterial activity against nonresistant bacterial strains [40].

Antimicrobial peptides (AMPs) are an interesting class of antibiotics. Compared with conventional antibiotics, they have unique antibiotic activities and low-resistance development trends. AMPs and peptides containing AMP conjugates have increasingly become the focus of research [41]. Anwar et al. reported that the combination of gold nanoparticles led to enhanced biological activity of nystatin and fluconazole (a clinically available drug for the treatment of amoeba and fungal infections) and amphotericin B, which plays an important role in the treatment of fungal infections [42]. Patra et al. reported that when administered together with the standard antibiotics kanamycin and rifampicin, synthesized gold nanoparticles showed potential antibacterial activity against five food-borne pathogens and demonstrated high synergistic activity. They also showed antioxidant and antiprotease inhibitory potential [43]. Yang et al. proposed gold nanoparticles (AuNPs) based on polyvalent amino sugars to treat wounds infected by super bacteria. Using dglucosamine (GluN) to modify gold nanoparticles (AuNPs), AuNPs based on polyvalent amino sugars were synthesized. $\mathrm{Au}$ _GluN does not damage the red blood cells outside the body but also has excellent super bacteria infection wound healing ability [44].

3.2.2. Joint Antibacterials. Ampicillin functionalized AuNP prepared by Brown et al. was an effective broad-spectrum fungicide that can resist Gram-negative and Gram-positive bacteria. This functionalization method mainly utilizes ampicillin's ability to permeate the outer membrane of bacteria. Nanoparticles enter the bacteria to achieve antibacterial effects. This combined antibacterial effect can destroy ampicillin-resistant bacteria [29]. Chavan et al. synthesized ampicillin-terminated gold nanoparticles (Amp-Au NPs) and studied their interaction with bacterial cells. In this synthesis process, the primary amine group of ampicillin acts as both a reducing agent and a capping agent. The nanogold not only maintains high antibacterial properties against ampicillin-sensitive bacteria but also proves to be effective against ampicillin-resistant bacteria [45]. Wang et al. used a sulfur-gold ( $\mathrm{S}-\mathrm{Au}$ ) bond to connect a cysteine-modified antimicrobial peptide and the broad-spectrum antimicrobial agent triclosan (TCS) to gold nanoparticles to prepare new types of antimicrobial peptide/triclosan double modified gold nanoparticles combined with antibacterial agents, improving the therapeutic effects and reducing the amount of antibacterial drugs, thereby decreasing the drugs' side effects. In an antibacterial experiment with vancomycin-resistant golden Staphylococcus aureus, double-modified gold nanoparticles showed better antibacterial properties than single-modified and unmodified gold nanoparticles due to the peptides' antibacterial ability to destroy bacterial cell walls and the antibacterial effect of TCS [35]. Some studies have shown that the combination of AuNPs, cefotaxime, and ciprofloxacin has an effect on all Salmonella bacteria. AuNPs destroy the homeostasis of cations, while conventional antibiotics promote the accumulation of reactive oxygen species, which leads to bacterial cell death. This process also induces apoptosis of Salmonella cells [46]. Li et al. reported that cationic and hydrophobic functionalized gold nanoparticles can effectively inhibit the growth of 11 clinical MDR isolates, including Gram-negative and Gram-positive bacteria, for longterm resistance to MDR bacteria (the key question in health care), providing a promising strategy [3]. Badwaik et al. studied the antibacterial properties of dextrose-coated gold nanoparticles (dGNPs). Experiments showed that dGNPs have bacteriostatic and bactericidal effects and are effective against both Gram-negative and Gram-positive bacteria [47]. Inbaraj et al. synthesized gold nanoparticles capped with chitosan (CH-NGs), ethylene glycol chitosan (GC-NGs), and poly $(\gamma$ glutamic acid) (PA-NGs) and characterized and evaluated its catalytic and antibacterial activity. The three synthetic functionalized gold nanoparticles all have a certain antibacterial effect [48]. Wang et al. used daptomycin (Dap) micelles as a template and reducing agent to prepare stable daptomycin golden nanoflowers (Dap-Aun NFs) under mild conditions. Under $808 \mathrm{~nm}$ near-infrared light radiation, Dap-Au 6 NFs inhibited the growth of tumors and bacteria [49].

3.2.3. Antibacterial Materials. Many special-purpose materials demonstrate certain antibacterial properties, so there are research options to use nanogold to achieve this goal. Shi and Zhuang used attapulgite as a carrier to obtain nanogold/attapulgite (Au-AOAT) with a controlled particle size and uniform distribution by reducing chloroauric acid using sodium borohydride prepared by adsorbing methylene blue on Au-AOAT. The antibacterial properties of nanogold effectively solved the problem that polymethyl methacrylate (PMMA), a medical material commonly used in the body, can easily cause bacterial infections in the body's dark environment [50]. Duan's antibacterial viscose fiber prepared by in situ synthesis combined with gold nanoparticle viscose fiber showed excellent antibacterial performance and stability and has application prospects in the fields of medicine and health [51]. MacDonald et al. used a simple procedure with thiol-terminated gold nanoparticles to develop an antibacterial surface that effectively killed bacteria in dark and bright conditions and showed that the method was effective under a variety of conditions, which opens up more possibilities for different technologies and new antibacterial coating applications [52]. Xie et al. proposed to adjust the antibacterial spectrum of DGNP (DAPT-GNP, DGNP) modified by small molecules (4,6-diamino-2-pyrimidinethiol, DAPT) by adjusting the size of small molecules. Ultrasmall DGNPs (uDGNPs) $(<2 \mathrm{~nm})$ show a broad antibacterial spectrum, especially the antibacterial efficacy against Gram-positive $(\mathrm{G}+)$ bacteria increased by more than 60 times. uDGNPfunctionalized scaffolds (agarose gel) can be used as a general wound dressing for curing burn infection [53].

3.2.4. Antibacterial Effects of Photothermal Effect. With the emergence of multidrug-resistant bacteria, photothermal therapy has been proposed as an alternative to antibiotics that target and kill pathogens [54]. Fu et al. modified synthesized peptides A and B on the surface of gold nanoparticles (GNPs) via Au-S bond reactions and then mixed them in 
equal proportions to form a GNP system. The prepared GNP system generated responsive aggregation in a weak acid environment of simulated bacteria, and large-sized aggregates that formed significantly improved its light-to-heat conversion efficiency. In vitro and bacterial mixed solutions were then produced under laser irradiation. When the temperature rapidly increased to $70^{\circ} \mathrm{C}$, the specific photothermal treatment of bacteria proved to have efficient antibacterial ability [55]. Millenbaugh et al. combined antibodies specific for Staphylococcus aureus with peptidoglycan and gold nanoparticles and exposed them to pulsed laser radiation, so that the functionalized nanoparticles were targeted to specific biological treatments and had a bactericidal effect. It had a linear relationship with the laser energy density and can be used alone or as an adjunct to existing conventional antibiotic treatment [56]. Studies indicated that hydrophilic functionalized polyethylene glycol-gold nanorods and hydrophobic functionalized polystyrene-gold nanorods can enhance antibacterial activity under the action of photopyrolysis [57]. A new type of photoactivatable nano antibiotic platform (TCPCM @ GNC-PND) was constructed by combining gold nanocages (GNC) and two thermal gates. Due to the photothermal effect induced by NIR and the synergistic effect of chemotherapeutic drugs, both in vitro and in periodontitis models can be effectively killed bacteria with little toxicity [58]. Peng et al. proposed an antibacterial strategy using bacteriophages combined with AuNRs. The phage attaches to the target bacteria, and NIR light irradiates the nanorods to cause LSPR excitation. This energy is released in the form of heat, destroying bacteriophages and bacteria that bind to the bacteriophages [59]. The Au-LTSL-GA.Au nanoparticles designed by Zhang et al. are used as nanophotosensitizers, based on the phototherapeutic sensitization properties of $\mathrm{Au}$ nanorods (NRs) and the antitumor activity of GA.A, to achieve synergistic photochemotherapy. In the antibacterial experiment, Au-LTSL-GA.A + NIR radiation has a broadspectrum antibacterial effect, and it has strong antibacterial activity against resistant E. coli and Staphylococcus aureus [60]. Rivas Aiello et al. analyzed the antibacterial effects of riboflavin (Rf) and pectin-coated gold nanoparticles (PecAuNP) on Staphylococcus aureus (S. aureus) and Pseudomonas aeruginosa (P. aeruginosa) as appropriate. In the photodynamic inactivation strategy of microorganisms, microbiological analysis showed that the presence of PecAuNP enhanced the antibacterial activity of lightirradiated Rf against Staphylococcus aureus and Pseudomonas aeruginosa [61].

3.2.5. Other Methods. Some scholars studied the photothermal-induced bactericidal activity of phospholipiddecorated gold nanorod (DSPE-AuNR) suspensions of Pseudomonas aeruginosa plankton membranes and biofilm cultures. The results showed that the gold nanorods coated with phospholipids were a promising nanoplatform [62]. Hayden et al. reported that cationic monolayer-protected gold nanoparticles can interact with the cell membranes of E. coli and Bacillus subtilis, forming distinctly different gold nanoparticle surface aggregation patterns or cell lysis depending on the size of the gold nanoparticles [63]. By using a cationic ligand (11-mercaptodecyl)-N,N,N-trimethylammonium bromide (MUTAB), Li et al. proved that water-soluble, positively charged AuNCs can be easily synthesized in a one-step reaction. These positively charged MUTAB-AuNCs show effective antibacterial activity against both Gramnegative and Gram-positive bacteria without causing drug resistance, including multidrug-resistant (MDR) bacteria and clinical bacteria [64]. It has been reported that lowtemperature plasma treatment can effectively kill Streptococcus mutans, and when used in combination with gold nanoparticles, it enhances the killing effect of lowtemperature plasma on Streptococcus mutans [65]. Goldloaded cerium oxide nanoparticles may be potential nanomaterials for in vivo applications due to their biocompatibility and antibacterial properties [66]. Wang et al. reported that two groups with different binding affinity to gold were used as anchor groups, and thiols or amines were used to modify phenylboronic acid on gold nanoparticles (AuNPs) with different densities. Different bacteria will have selective antibacterial properties, which are expected to be used for personalized treatment [67]. Gold nanoclusters conjugated with various surface ligands have high biocompatibility, multivalent effects, easy modification, and photothermal stability, so they are potential antibacterial agents. Gold nanoclusters can carry different charges and even have target specificity for bacteria, but their antibacterial activity still needs to be improved to meet clinical needs [68]. Jalil et al. conducted a systematic study on the changes in the surface structure of gold ( $\mathrm{Au}$ ) induced by femtosecond laser and its hydrophobicity and bacterial adhesion characteristics. It proves that the formed surface structure can reduce the adhesion of E. coli bacteria and shows that fs-LIPSSs enjoy excellent antibacterial adhesion performance due to its large-scale surface coverage. Approximately $99.03 \%$ of fs-LIPSSs have no bacteria attached to the surface [69]. Pišlová et al. added nanogold to liquid polyethylene glycol (PEG). PEG is used as the basic biocompatible stabilizer of AuNP colloid. In addition, two naturally occurring polysaccharides, chitosan (Ch) and methyl cellulose (MC), were separately diluted into a PEG matrix. Two bacterial strains (Gram-positive Staphylococcus epidermidis and Gram-negative Escherichia coli) were tested for antibacterial and proved to have certain antibacterial effects [70]. Simon et al. studied the antibacterial activity of bovine serum albumin (BSA), human serum albumin (HSA), and egg white lysozyme (HEWL) with gold nanoparticles (GNP) against four pathogens. This study proposes a new method to regulate the antibacterial activity of HEWL against Pseudomonas aeruginosa, in order to solve the problem of drug resistance of this pathogen in chemotherapy for many diseases [71].

\section{Functionalized Gold Nano- Antibacterial Mechanisms}

Many functional modification methods are used to produce gold nanoparticles with antibacterial properties (as summarized in Figure 1). The effect mechanisms of gold nanoparticles prepared using different methods on bacteria also vary. 


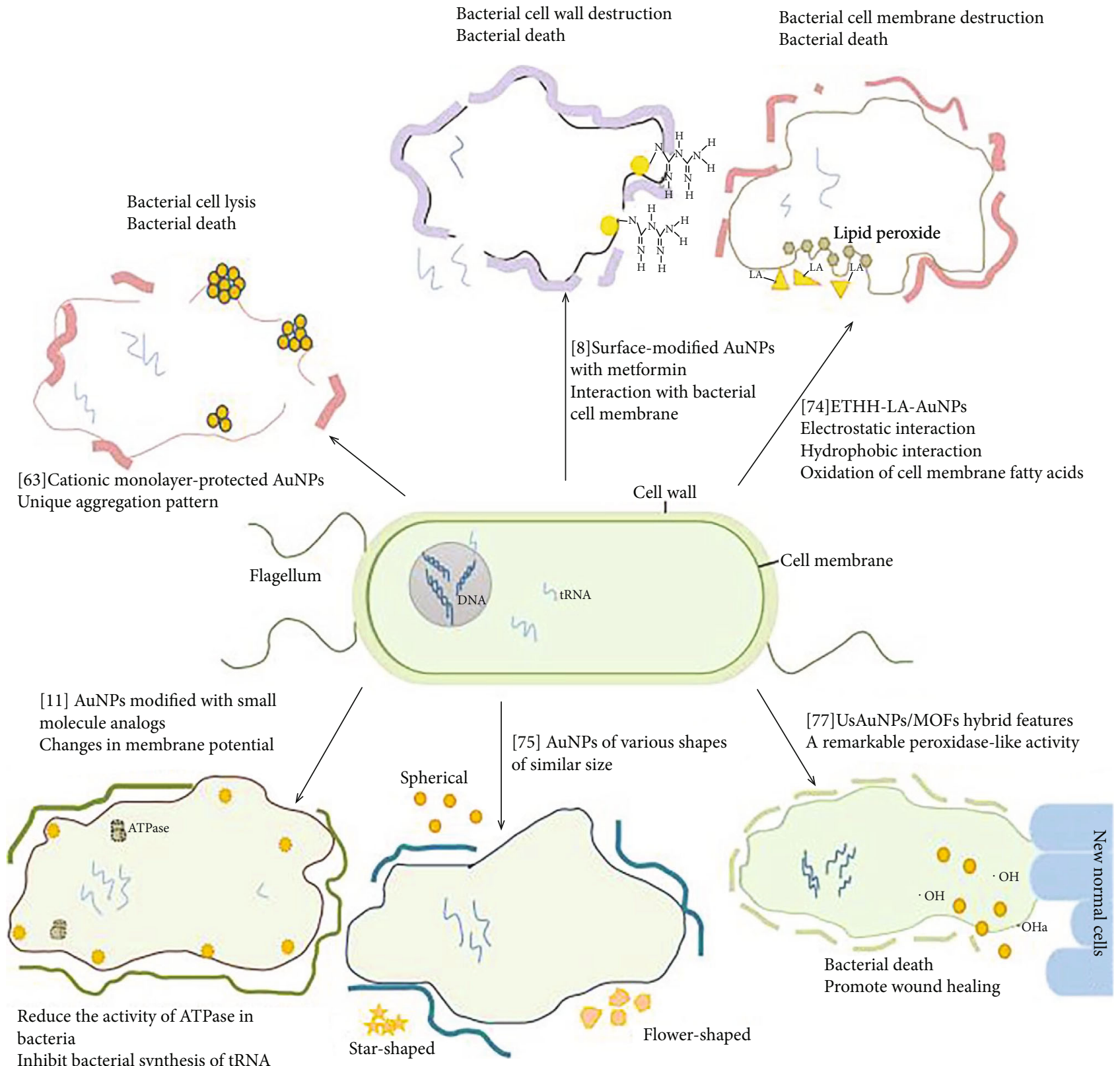

Bacterial death

Figure 1: A brief summary of the antibacterial mechanisms and effects of 6 functionalized gold nanoparticles.

The specific antibacterial mechanisms of gold nanoparticles are also unclear.

4.1. Function with Cell Membranes. Types of modified gold nanoparticles can interact with bacterial cell membranes, destroying their integrity and playing an antibacterial and sterilizing role [4]. Studies reported that AuNPs protected by $2 \mathrm{~nm}$ core cationic monolayers can interact with the cell membranes of Gram-positive and Gram-negative bacteria, forming unique aggregation patterns and bacterial cell lysis, indicating that the cationic surface properties of $2 \mathrm{~nm}$ AuNPs can be used as antibacterial agents [63]. Li et al. confirmed that cationic and hydrophobic functionalized AuNPs can effectively inhibit the growth of 11 clinical MDR isolates, including Gram-negative and Gram-positive bacteria [3]. Ahmady et al. used aryl gold diazonium (iii) salt [HOOC4-C6H4N[triple bond, length as m-dash]N] to reduce $\mathrm{AuCl} 4$ green and conjugate the resulting AuNPs-C6H4-4-COOH with lysozyme together for antibacterial [72].

4.2. Combination with Drugs. Jiang et al. found that when mercaptopyrimidine molecules with no antibacterial activity were modified on the surface of gold particles, gold nanoparticles showed good antibacterial activity against Gramnegative bacteria and multidrug-resistant negative bacteria. These types of nonantibiotic drugs and gold nanoparticles 
coated with mercaptopyrimidine can produce broadspectrum antibacterial and bactericidal effects against super bacteria. Metformin shows the best enhancement activity by improving the ability to destroy the bacterial cell wall [8]. Khosravi et al. evaluated the antibacterial, antiefflux, antibiofilm, and antimucus (extracellular polysaccharide) effects of AuNPs synthesized from Anthemis atropatana extract on multidrug-resistant (MDR) Klebsiella pneumoniae. The new therapies they developed demonstrate the potential use of AuNPs in preventing biofilm-related Klebsiella pneumoniae infections [73].

4.3. Electrostatic and Hydrophobic Interactions. Ranjan Sarker et al. synthesized elongated tetrahedral (ETHH) $\mathrm{Au}$ nanoparticles functionalized with lipoic acid (LA), a natural antioxidant with terminal carboxylic acid and dithiolane rings, to produce ETHH-LA Au nanoparticles. The results showed that LA enhanced the antibacterial activity of the ETHH Au nanoparticles. The Au nanoparticles interacted with bacteria through electrostatic and hydrophobic interactions, destroying bacterial cell walls and subsequently oxidizing fatty acids in cell membranes [74].

4.4. Changing the Membrane Potential. Tian et al. used small molecule analogs in bacteria as drug precursors and modified them by attaching them to the surface of gold particles. Through genomic and proteomic analysis of bacteria treated with gold nanoparticles, gold nanoparticles were achieved through two antibacterial effect mechanisms: one reduced the activity of ATPase in bacteria by changing the membrane potential, and the other inhibited the synthesis of tRNA by bacteria [11].

4.5. Changing Its Shape. A study found that the effect of different forms of gold nanoparticles on antibacterial properties may also be related to its antibacterial mechanism. Penders et al. synthesized gold nanoparticles (AuNPs) of various shapes (including spherical, star-shaped, and flower-shaped) of similar size and evaluated their antibacterial effects on Staphylococcus aureus. It shows that AuNFs may be suitable for use as a new antibacterial agent (without antibiotics). By using the plasmon resonance effect of AuNPs, the antibacterial effect can be further enhanced [75]. Hameed et al. studied the antibacterial effects of nanospheres (AuNSps), nanostars (AuNSts), and nanocubes (AuNCs) on Escherichia coli, Pseudomonas aeruginosa, and Staphylococcus aureus at lower concentrations. The conclusion drawn from this study is that AuNPs show significant antibacterial properties at lower concentrations [76].

4.6. As Nanozyme. Hu et al. grew ultrafine gold nanoparticles (UsAuNPs) on ultrathin two-dimensional metal organic frameworks (MOF) by in situ reduction. The formed UsAuNP/MOF hybrid has the advantages of UsAuNPs and ultrathin 2D MOF and exhibits significant peroxidase-like activity for the decomposition of $\mathrm{H} 2 \mathrm{O} 2$ into toxic hydroxyl radicals $(\mathrm{OH})$. The results show that the prepared UsAuNP/MOF nanozymes have excellent antibacterial properties against both Gram-negative bacteria (E. coli) and Grampositive bacteria (Staphylococcus aureus) with the aid of low-dose $\mathrm{H}_{2} \mathrm{O}_{2}$. Animal experiments show that this hybrid material can effectively promote wound healing and has good biocompatibility [77]. Brasili et al. adsorbed lysozyme onto gold nanoparticles and performed self-limiting assembly based on plaque-like plasmon colloids, thereby obtaining a new type of antibacterial nanomaterial. It has also been proved that the change of its catalytic activity will lead to the enhancement of the antibacterial performance of the appropriately sized components [78].

\section{Application of Gold Nanoparticles in Oral Biology}

Oral microflora represent an important part of the human microbiome, including thousands of species. They are normal components of the oral environment and have an important function to prevent the colonization of foreign bacteria, thereby affecting overall health. The most common oral diseases dental caries, gingivitis, and periodontitis are also based on microorganisms [79]. At present, antibiotics are used in dental practice, dental treatment, and infection prevention, but the indications for using systemic antibiotics in the dental field are limited. The characteristics of using antibiotics in dental practice are based on empirical prescriptions of clinical and bacterial epidemiological factors, resulting in the use of a very narrow range of broad-spectrum antibiotics in a short period of time. This has led to the development of drug resistance (AMR) in many microorganisms and the ineffectiveness of commonly used antibiotics. Antibiotic resistance and other side effects of antibiotic abuse have recently increased [80]. Although many studies have focused on developing antibacterial drugs to overcome these problems, most attempts have failed to achieve the desired results. Nanomaterials are promising in antibacterial therapy due to their enhanced and unique physical and chemical properties (such as ultrasmall size, large surface area/mass ratio, and increased chemical reactivity). Nanoparticles (NPs) may provide a new strategy for treating and preventing dental infections. Metal and organic NPs have been used in many dental fields due to their broad-spectrum bactericidal properties [81]. In particular, gold nanomaterials have been extensively studied due to their high stability, amazing chemical and physical properties, and antibacterial properties [82]. With the continuous research into gold nanoparticles, many scholars have begun to study their application in oral biology. The following is a brief introduction to the application of gold nanoparticles in oral biology. The antibacterial 4,6-diamino-2-pyrimidinethiol-modified gold nanoparticles (AuDAPT) newly developed by Zhang et al. were coated on the alignment agent. The coated aligning agent shows good antibacterial activity against Porphyromonas gingivalis. In the presence of the coated aligning agent, the number of planktonic cells is reduced and the formation of biofilms is prevented. This material also exhibits good biocompatibility in vivo and in vitro [83]. Tetracycline antibiotics (TCs) are a broad-spectrum antibacterial agent. However, excessive use of TC poses a threat to the environment and human health and causes tetracycline teeth in the oral cavity. Therefore, it is necessary to develop a simple method 
for detecting TC. Wu et al. designed a new sensor based on gold nanoparticle (AuNP) aggregation and coordinated control of ss-DNA fragments to detect multiple antibiotics. The sensor has excellent selectivity and sensitivity for the separation and detection of TET and CAP $[84,85]$. Li et al. used Lhistidine-terminated gold nanoclusters (His-AuNCs) as FL indicators and $\mathrm{Eu} \mathrm{3+}$ FL enhancers. A simple method for selectively detecting TC is reported [86]. At the same time, it is not only for specific antibiotics. For commonly used antibiotics such as amoxicillin, chlortetracycline, and erythromycin, Leng et al. proposed a method of $\mathrm{Au}(\mathrm{I})$-mediated protein and antibiotic interconnection to generate multicolor gold nanoparticles (GNP). The method is used for colorimetric determination and differentiation of antibiotics [87]. Jadhav et al. evaluated the application of plant-mediated osteoinductive properties of gold nanoparticles in dental implants. Green synthetic gold nanoparticles showed good stability and osteoinductive potential in various blood components ( $0.2 \mathrm{M}$ of histidine, $0.2 \mathrm{M}$ of cysteine, $2 \%$ bovine serum albumin, and 2\% human serum albumin) [88]. Heo et al. reported that gold nanoparticles are very suitable as osteogenic agents because of their potential impact on stimulating osteoblast differentiation. In their study, titanium implants with bone fusion were coated with gold nanoparticles to promote bone regeneration. In vitro and in vivo tests demonstrated that $\mathrm{Ti}-$ GNPs can be used as an osseointegration-induced dental implants to form bone interfaces and maintain new bone formation [89]. Yang et al. developed a new type of calcium phosphate cement containing gold nanoparticles (GNP$\mathrm{CPC}$ ) to study its osteoinductive capacity for human dental pulp stem cells (hDPSCs). The results proved that GNPCPC significantly enhanced the osteogenic function of hDPSCs. GNP is expected to modify CPC through nanomorphology and function as a bioactive additive to enhance bone regeneration [90]. The development of multifunctional nanomaterials is one of the most interesting and advanced research fields in nanotechnology and is expected to revolutionize cancer diagnosis and treatment methods. The unique optical properties of gold nanoparticles and their utility in photothermal and radiotherapy have expanded a new platform for the early detection and treatment of cancer. Gold nanoparticles (AuNPs) exhibit good physical properties and tailor-made surface functionalization, providing a potential platform for the development of cancer therapeutics. GNPbased nanostructures are nontoxic and have large surface area biocompatibility, making it possible to modify their surfaces with various chemicals including different polymers, antibodies, and even drug molecules. They are used for targeted drug delivery to carry drugs and selectively release them into the desired tissue, reducing the damaging effect on healthy cells while increasing the dose of cancer drugs [91, 92]. Satapathy et al. formulated mixed nanoparticles (QAuNPs) using quinacrine and gold and characterized/studied their antiangiogenic and antimetastatic effects on OSCC-CSCs. In xenograft mouse models, QAuNPs significantly inhibited cell proliferation, caused apoptosis in vitro, and destroyed angiogenesis and tumor regression in vivo [93]. Durgesh et al. evaluated microbial contamination and plaque scores of gold nanoparticle-coated and uncoated toothbrushes. The results showed that toothbrushes coated with gold nanoparticles demonstrated significantly lower bristle contamination and plaque scores after one week compared to uncoated toothbrushes that did not use dentifrice [94]. Colombé et al. evaluated the potential of ultra-small gold $(\mathrm{Au})$ nanoclusters $(\mathrm{NCs})$ in optical image-guided surgery and used AuNCs as contrast agents for image-guided surgery of head and neck tumors. The results were compared with a control group. The survival time increased, and there was no local recurrence [95]. Kim et al. demonstrated that stimuli-responsive plasma gold nanoclusters (AuNCs) can be used as contrast agents for optical coherence tomography (OCT) to detect early stage cancer using molecularly programmable inorganic nanomaterials. The contrast agent has the potential to diagnose early cancer. The contrast agent can generate multiple stimulus-triggered diagnostic signals in early cancer [96]. Chakraborty et al. developed an ELISA system based on gold nanoparticles to detect noninvasive osteopontin (overexpressed in oral squamous cell carcinoma). The incorporation of gold nanorods or gold nanospheres using conventional ELISA can increase the sensitivity of the analysis. The detection limits of gold nanorods (detection limit: $0.02 \mathrm{ng} \cdot \mathrm{mL}^{-1}$ ) and gold nanospheres (detection limit: $0.03 \mathrm{ng} \cdot \mathrm{mL}^{-1}$ ) decreased significantly. Compared with commercially available OPN ELISA kits, an improved detection method (detection limit) was obtained $\left(0.14 \mathrm{ng} \cdot \mathrm{mL}^{-1}\right)$. The improved ELISA had a wide linear detection range $\left(0.31-20 \mathrm{ng} \cdot \mathrm{mL}^{-1}\right)$ and good reproducibility and specificity for the tested interfering substances in saliva [97]. Li et al. reported that gold nanoparticles can promote the proliferation of human periodontal ligament stem cells [98]. Daood et al. reported that using TPAu and EDC to cross-link dentin collagen through TPAu/EDC nanoparticle formulations improved the antibiodegradability of demineralized dentin matrix, inhibited protease, and increased the mechanical and structural stability [99].

\section{Prospects of Gold Nanoparticles}

Junevičius et al. used toothpaste containing silver and gold particles and toothpaste containing zinc citrate as active ingredients and 8 standard microbial cultures to evaluate the antibacterial activity of each study formulation at 9 different concentrations. The results showed that gold was not as effective as silver [100]. Although silver nanoparticles are considered promising antibacterial agents due to their antibacterial activity, the acute cytotoxicity of silver ions released by silver nanoparticles may limit their potential practical applications. Zhu et al. found that compared with Ag nanoplates, irradiated porous $\mathrm{Ag}-\mathrm{Au}$ nanoplates have similar antibacterial ability to Staphylococcus aureus strains and have lower cytotoxicity in vitro [101]. Moreover, studies by Chlumsky et al. found that even if AuNPs, AgNPs, and CS and their combinations could not remove biofilms, they also reduced the metabolic activity of all tested strains by at least $80 \%$ [102]. The experimental results of Saravanakumar and others proved the multifunctional biological activities of CS-AuNPs, including antioxidant, antibacterial, antidiabetic and anticancer activities, which are worthy of further 
research [103]. The nanoparticles' different sizes and surface chemistry regulated cell uptake and nanoparticle toxicity, adversely affecting their clinical application [104]. Studies have demonstrated that nanoparticles are removed by the mononuclear phagocytic system. Particles with different shapes will influence their distribution on target organs, ultimately affecting the particles' biological toxicity, but no significant sublethal effects have been observed [105]. Due to their small size, nanoparticles can transfer to the systemic circulation after skin contact, inhalation, or oral administration. Once in the bloodstream, nanoparticles come into contact with different blood components and may interfere with normal platelet function, causing bleeding or thrombosis. The compatibility of nanoparticles with blood elements remains a controversial topic [106]. Overcoming multidrug resistance (MDR) has become the direction of improving antibiotics, and nanodrug delivery systems have opened up new ways for this. Riaz et al. investigated the effect of flavonoidcoated gold nanoparticles (FAuNPs) on the colonization of Enterococcus faecalis in the liver and kidney of mice. Compared with the use of free flavonoids, the bacterial count in mouse organs was significantly reduced [107]. Gold nanoparticles have many advantages such as simple and controlled synthesis, small size, and high biocompatibility and surface plasmon resonance, but whether they can be affected by in vivo or intracellular factors needs to be elucidated. Gold nanoclusters' small size and gold nanoparticles' different shapes improve their antibacterial effects, but further research is necessary on the toxicity of organisms after a long time and a large dose. It is also indispensable to study the influence or changes in the use of gold nanoparticles in a specific period in organisms or a defined physiological state in certain diseases. Gold nanoparticles are easily modified, and functionalized gold nanoparticles have considerable antibacterial application potential. Gold nanoparticles can be modified to improve their antibacterial properties for clinical antibacterial use. They can also be applied as drug carriers to improve drug efficacy, and their photothermal properties can be harnessed to kill bacteria. Combining gold nanoparticles with different materials produces drugs with antibacterial properties. In summary, gold nanoparticles' various characteristics can be used to functionalize them for specific requirements to provide new methods of solving different antibacterial challenges.

\section{Data Availability}

The data supporting this review are from previously reported studies and datasets, which have been cited.

\section{Conflicts of Interest}

The authors declare that there is no conflict of interest.

\section{Authors' Contributions}

Chen Su and Kun Huang contributed equally.

\section{Acknowledgments}

This work was financially supported by the Key Laboratory Foundation of Stomatology of Fujian Province University (Grant number: 2019kq03) and Fujian Provincial Program of Innovation and Entrepreneurship for Undergraduates (Grant number: S201910392030).

\section{References}

[1] G. M. Rossolini, F. Arena, P. Pecile, and S. Pollini, "Update on the antibiotic resistance crisis," Current Opinion in Pharmacology, vol. 18, pp. 56-60, 2014.

[2] Y. Zhao, Y. Tian, and X. Jiang, "Investigation of antibacterial activities of gold nanoparticles against Gram-positive bacteria," in Proceedings of the International Symposium on Quality Control of Biomaterials and Tissue Engineering Products., pp. 95-102, 2011.

[3] X. Li, S. M. Robinson, A. Gupta et al., "Functional gold nanoparticles as potent antimicrobial agents against multidrug-resistant bacteria," ACS Nano, vol. 8, no. 10, pp. 10682-10686, 2014.

[4] G. Chu and Y. Chen, "Research progress of antibacterial mechanism and application of nano-gold," Journal of Shanghai Jiaotong University, vol. 11, pp. 1386-1390, 2018.

[5] G. V. Vimbela, S. M. Ngo, C. Fraze, L. Yang, and D. A. Stout, "Antibacterial properties and toxicity from metallic nanomaterials," International Journal of Nanomedicine, vol. 12, pp. 3941-3965, 2017.

[6] S. Ashraf, B. Pelaz, P. del Pino et al., "Gold-based nanomaterials for applications in nanomedicine," Topics in Current Chemistry, vol. 370, pp. 169-202, 2016.

[7] R. A. Bapat, T. V. Chaubal, S. Dharmadhikari et al., "Recent advances of gold nanoparticles as biomaterial in dentistry," International Journal of Pharmaceutics, vol. 586, 2020.

[8] X. Jiang and Y. Jia, "Adjust the surface modification of gold nanoparticles to adjust their antibacterial properties," in Proceedings of the Chinese Chemical Society Conference, pp. 142$143,2016$.

[9] X. Li, "A study on toxicity and antimicrobial effects of Nano $\mathrm{Au}$," in Proceedings of the National Health Industry Enterprise Management Association Antibacterial Industry Branch Conference, pp. 172-187, 2006.

[10] S. Chatterjee, A. Bandyopadhyay, and K. Sarkar, "Effect of iron oxide and gold nanoparticles on bacterial growth leading towards biological application," Journal of Nanobiotechnology, vol. 9, no. 1, p. 34, 2011.

[11] Y. Tian, Y. Zhao, Y. Cui, and X. Jiang, "The synthesis of gold nanoparticles with activities against multidrug-resistant bacteria and mechanism of action," Proceedings of the Chinese Chemical Society Conference, , 2012.

[12] S. S. I. Abdalla, H. Katas, F. Azmi, and M. F. M. Busra, “Antibacterial and anti-biofilm biosynthesised silver and gold nanoparticles for medical applications: mechanism of action, toxicity and current status," Current Drug Delivery, vol. 17, no. 2, pp. 88-100, 2020.

[13] C. Carnovale, G. Bryant, R. Shukla, and V. Bansal, "Size, shape and surface chemistry of nano-gold dictate its cellular interactions, uptake and toxicity," Progress in Materials Science, vol. 83, pp. 152-190, 2016. 
[14] Á. I. López-Lorente, S. Cárdenas, and Z. I. González-Sánchez, "Effect of synthesis, purification and growth determination methods on the antibacterial and antifungal activity of gold nanoparticles," Materials Science and Engineering: C, vol. 103, 2019.

[15] A. Rónavári, N. Igaz, M. K. Gopisetty et al., "Biosynthesized silver and gold nanoparticles are potent antimycotics against opportunistic pathogenic yeasts and dermatophytes," International Journal of Nanomedicine, vol. 13, pp. 695-703, 2018.

[16] M. P. Patil and G. D. Kim, "Eco-friendly approach for nanoparticles synthesis and mechanism behind antibacterial activity of silver and anticancer activity of gold nanoparticles," Applied Microbiology and Biotechnology, vol. 101, no. 1, pp. 79-92, 2017.

[17] I. Fierascu, M. I. Georgiev, A. Ortan et al., "Phyto-mediated metallic nano-architectures via Melissa officinalis L.: synthesis, characterization and biological properties," Scientific Reports, vol. 7, no. 1, article 12428, 2017.

[18] V. D. Doan, V. S. Luc, T. L. H. Nguyen, T. D. Nguyen, and T. D. Nguyen, "Utilizing waste corn-cob in biosynthesis of noble metallic nanoparticles for antibacterial effect and catalytic degradation of contaminants," Environmental Science and Pollution Research International, vol. 27, no. 6, pp. 6148-6162, 2020.

[19] Y. C. Wang, É. Rhéaume, F. Lesage, and A. Kakkar, "Synthetic methodologies to gold nanoshells: an overview," Molecules, vol. 23, no. 11, p. 2851, 2018.

[20] S. Zhu, Y. Shen, Y. Yu, and X. Bai, "Synthesis of antibacterial gold nanoparticles with different particle sizes using chlorogenic acid," Royal Society Open Science, vol. 7, no. 3, 2020.

[21] P. S. Murphin Kumar, D. MubarakAli, R. G. Saratale et al., "Synthesis of nano-cuboidal gold particles for effective antimicrobial property against clinical human pathogens," Microbial Pathogenesis, vol. 113, pp. 68-73, 2017.

[22] S. G. Ali, M. A. Ansari, M. A. Alzohairy et al., "Biogenic gold nanoparticles as potent antibacterial and antibiofilm nanoantibiotics against Pseudomonas aeruginosa," Antibiotics, vol. 9, no. 3, p. 100, 2020.

[23] L. Mocan, I. Ilie, C. Matea et al., "Surface plasmon resonanceinduced photoactivation of gold nanoparticles as bactericidal agents against methicillin-resistant Staphylococcus aureus," International Journal of Nanomedicine, vol. 9, pp. 14531461, 2014.

[24] P. Singh, S. Pandit, J. Garnæs et al., "Green synthesis of gold and silver nanoparticles from Cannabis sativa (industrial hemp) and their capacity for biofilm inhibition," International Journal of Nanomedicine, vol. 13, pp. 3571-3591, 2018.

[25] M. G. Arafa, R. F. El-Kased, and M. M. Elmazar, “Thermoresponsive gels containing gold nanoparticles as smart antibacterial and wound healing agents," Scientific Reports, vol. 8, no. 1, article 13674, 2018.

[26] Y. Zhang, T. P. Shareena Dasari, H. Deng, and H. Yu, "Antimicrobial activity of gold nanoparticles and ionic gold," Journal of Environmental Science and Health Part C: Environmental Carcinogenesis \& Ecotoxicology Reviews, vol. 33, no. 3, pp. 286-327, 2015.

[27] G. L. Burygin, B. N. Khlebtsov, A. N. Shantrokha, L. A. Dykman, V. A. Bogatyrev, and N. G. Khlebtsov, "On the enhanced antibacterial activity of antibiotics mixed with gold nanoparticles," Nanoscale Research Letters, vol. 4, no. 8, pp. 794-801, 2009.
[28] J. N. Payne, H. K. Waghwani, M. G. Connor et al., "Novel synthesis of kanamycin conjugated gold nanoparticles with potent antibacterial activity," Frontiers in Microbiology, vol. 7, 2016.

[29] A. N. Brown, K. Smith, T. A. Samuels, J. Lu, S. O. Obare, and M. E. Scott, "Nanoparticles functionalized with ampicillin destroy multiple-antibiotic-resistant isolates of Pseudomonas aeruginosa and Enterobacter aerogenes and methicillin-resistant Staphylococcus aureus," Applied and Environmental Microbiology, vol. 78, no. 8, pp. 2768-2774, 2012.

[30] K. Alsamhary, N. Al-Enazi, W. A. Alshehri, and F. Ameen, "Gold nanoparticles synthesised by flavonoid tricetin as a potential antibacterial nanomedicine to treat respiratory infections causing opportunistic bacterial pathogens," Microbial Pathogenesis, vol. 139, 2020.

[31] M. A. Mohamady Hussein, F. G. D. Baños, M. Grinholc, A. S. Abo Dena, I. M. El-Sherbiny, and M. Megahed, "Exploring the physicochemical and antimicrobial properties of goldchitosan hybrid nanoparticles composed of varying chitosan amounts," International Journal of Biological Macromolecules, vol. 162, pp. 1760-1769, 2020.

[32] L. A. Dykman and N. G. Khlebtsov, "Gold nanoparticles in chemo-, immuno-, and combined therapy: review [Invited]," Biomedical Optics Express, vol. 10, no. 7, pp. 3152-3182, 2019.

[33] S. Govindaraju, M. Ramasamy, R. Baskaran, S. J. Ahn, and K. Yun, "Ultraviolet light and laser irradiation enhances the antibacterial activity of glucosamine-functionalized gold nanoparticles," International Journal of Nanomedicine, vol. 10, pp. 67-78, 2015.

[34] M. Bajaj, S. K. Pandey, T. Nain et al., "Stabilized cationic dipeptide capped gold/silver nanohybrids: towards enhanced antibacterial and antifungal efficacy," Colloids and Surfaces B: Biointerfaces, vol. 158, pp. 397-407, 2017.

[35] J. Wang, X. Cai, L. Yang, F. Huang, and J. Liu, “Antibacterial peptide/triclosan dual functionalized gold nanoparticles for enhanced antibacterial effect," Biomedical Engineering and Clinical Medicine, vol. 3, pp. 252-257, 2019.

[36] M. S. Jabir, A. A. Taha, and U. I. Sahib, "Linalool loaded on glutathione-modified gold nanoparticles a drug delivery system for a successful antimicrobial therapy," Artificial Cells, Nanomedicine, and Biotechnology, vol. 46, Supplement 2, pp. 345-355, 2018.

[37] J. Lin, Z. He, Y. Zhou, and H. Deng, "Mesoporous silica coated $\mathrm{Au}$ nanobipyramids nanoparticle for nearinfrared responsive drug delivery system and its application in antibacteria," Stomatology, vol. 5, pp. 404-408, 2019.

[38] Y. Zhao and X. Jiang, "Gold nanoparticles activating small molecules as antibacterial agents," Proceedings of the Conference of the Chinese Society of Biomedical Engineering, vol. 214, 2010.

[39] X. Cao, X. Wang, H. Dong, X. Wang, and B. Liu, "Study on the promotion of nano-gold on sonodynamic antibacterial chemotherapy," in Proceedings of the National Health Industry Enterprise Management Association Antibacterial Industry Branch Conference, pp. 193-194, 2018.

[40] S. Kalita, R. Kandimalla, A. C. Bhowal, J. Kotoky, and S. Kundu, "Functionalization of $\beta$-lactam antibiotic on lysozyme capped gold nanoclusters retrogress MRSA and its persisters following awakening," Scientific Reports, vol. 8, no. 1, article 5778, 2018. 
[41] A. Reinhardt and I. Neundorf, "Design and application of antimicrobial peptide conjugates," International Journal of Molecular Sciences, vol. 17, no. 5, p. 701, 2016.

[42] A. Anwar, R. Siddiqui, M. Shah, and N. Khan, "Gold nanoparticles conjugation enhances antiacanthamoebic properties of nystatin, fluconazole and amphotericin B," Journal of Microbiology and Biotechnology, vol. 29, no. 1, pp. 171-177, 2019.

[43] J. K. Patra and K. H. Baek, "Novel green synthesis of gold nanoparticles using Citrullus lanatus rind and investigation of proteasome inhibitory activity, antibacterial, and antioxidant potential," International Journal of Nanomedicine, vol. 10, pp. 7253-7264, 2015.

[44] X. Yang, Q. Wei, H. Shao, and X. Jiang, "Multivalent aminosaccharide-based gold nanoparticles as narrowspectrum antibiotics in vivo," ACS Applied Materials \& Interfaces, vol. 11, no. 8, pp. 7725-7730, 2019.

[45] C. Chavan, S. Kamble, A. V. R. Murthy, and S. N. Kale, "Ampicillin-mediated functionalized gold nanoparticles against ampicillin-resistant bacteria: strategy, preparation and interaction studies," Nanotechnology, vol. 31, no. 21, article $215604,2020$.

[46] B. Lee and D. G. Lee, "Synergistic antibacterial activity of gold nanoparticles caused by apoptosis-like death," Journal of Applied Microbiology, vol. 127, no. 3, pp. 701-712, 2019.

[47] V. D. Badwaik, L. M. Vangala, D. S. Pender et al., "Sizedependent antimicrobial properties of sugar-encapsulated gold nanoparticles synthesized by a green method," Nanoscale Research Letters, vol. 7, no. 1, p. 623, 2012.

[48] B. S. Inbaraj, B. Y. Chen, C. W. Liao, and B. H. Chen, "Green synthesis, characterization and evaluation of catalytic and antibacterial activities of chitosan, glycol chitosan and poly $(\gamma$-glutamic acid) capped gold nanoparticles," International Journal of Biological Macromolecules, vol. 161, pp. 1484-1495, 2020.

[49] J. Wang, J. Zhang, K. Liu et al., "Synthesis of gold nanoflowers stabilized with amphiphilic daptomycin for enhanced photothermal antitumor and antibacterial effects," International Journal of Pharmaceutics, vol. 580, article 119231, 2020.

[50] R. Shi and W. Zhuang, "Preparation of nano-gold / attapulgite composite antibacterial agent adsorbing methylene blue and its application in PMMA," New Chemical Materials, vol. 4, 2017.

[51] T. Duan, "Preparation and properties of antibacterial viscose fiber," Progress in Textile Science \& Technology, vol. 7, pp. 79, 2019.

[52] T. J. Macdonald, K. Wu, S. K. Sehmi et al., "Thiol-Capped Gold Nanoparticles Swell-Encapsulated into Polyurethane as Powerful Antibacterial Surfaces Under Dark and Light Conditions," Scientific Reports, vol. 6, no. 1, article 39272, 2016.

[53] Y. Xie, J. Yang, J. Zhang, W. Zheng, and X. Jiang, “Activating Antibacterial Effect of 4,6-diamino-2-pyrimidinethiol-modified Gold Nanoparticles by Reducing their Sizes," Angewandte Chemie, 2020.

[54] T. T. Ngo-Duc, Z. Alibay, J. M. Plank, J. E. Cheeney, and E. D. Haberer, "Gold-Decorated M13 I-Forms and SForms for Targeted Photothermal Lysis of Bacteria," ACS Applied Materials \& Interfaces, vol. 12, no. 1, pp. 126-134, 2019.
[55] C. Fu, J. Chang, X. Jiang et al., "Responsive aggregation of gold nanoparticles system for photothermal therapy of bacteria in vitro," Acta Laboratorium Animalis Scientia Sinica, vol. 3, pp. 278-285, 2019.

[56] N. J. Millenbaugh, J. B. Baskin, M. N. DeSilva, W. R. Elliott, and R. D. Glickman, "Photothermal killing of Staphylococcus aureus using antibody-targeted gold nanoparticles," International Journal of Nanomedicine, vol. 10, pp. 1953-1960, 2015.

[57] N. N. Mahmoud, A. M. Alkilany, E. A. Khalil, and A. G. AlBakri, "Nano-Photothermal ablation effect of Hydrophilic and Hydrophobic Functionalized Gold Nanorods on Staphylococcus aureus and Propionibacterium acnes," Scientific Reports, vol. 8, no. 1, article 6881, 2018.

[58] L. Zhang, Y. Wang, C. Wang et al., "Light-Activable OnDemand Release of Nano-Antibiotic Platforms for Precise Synergy of Thermochemotherapy on Periodontitis," ACS Applied Materials \& Interfaces, vol. 12, no. 3, pp. 33543362, 2019.

[59] H. Peng, R. E. Borg, L. P. Dow, B. L. Pruitt, and I. A. Chen, "Controlled phage therapy by photothermal ablation of specific bacterial species using gold nanorods targeted by chimeric phages," Proceedings of the National Academy of Sciences of the United States of America, vol. 117, no. 4, pp. 19511961, 2020.

[60] W. Zhang, W. Yu, X. Ding et al., "Self-assembled thermal gold nanorod-loaded thermosensitive liposome-encapsulated ganoderic acid for antibacterial and cancer photochemotherapy," Artificial Cells, Nanomedicine, and Biotechnology, vol. 47, no. 1, pp. 406-419, 2019.

[61] M. B. Rivas Aiello, F. Ghilini, J. E. Martínez Porcel, L. Giovanetti, P. L. Schilardi, and D. O. Mártire, "Riboflavin-Mediated Photooxidation of Gold Nanoparticles and Its Effect on the Inactivation of Bacteria," Langmuir, vol. 36, no. 28, pp. 8272-8281, 2020.

[62] A. G. Al-Bakri and N. N. Mahmoud, "Photothermal-Induced Antibacterial Activity of Gold Nanorods Loaded into Polymeric Hydrogel against Pseudomonas aeruginosa Biofilm," Molecules, vol. 24, no. 14, article 2661, 2019.

[63] S. C. Hayden, G. Zhao, K. Saha et al., "Aggregation and interaction of cationic nanoparticles on bacterial surfaces," Journal of the American Chemical Society, vol. 134, no. 16, pp. 6920-6923, 2012.

[64] Y. Li, J. Zhen, Q. Tian et al., "One step synthesis of positively charged gold nanoclusters as effective antimicrobial nanoagents against multidrug-resistant bacteria and biofilms," Journal of Colloid and Interface Science, vol. 569, pp. 235243, 2020.

[65] S. R. Park et al., "Enhancement of the killing effect of lowtemperature plasma on Streptococcus mutans by combined treatment with gold nanoparticles," Journal of Nanobiotechnology, vol. 12, no. 1, article 29, 2014.

[66] K. S. Babu, M. Anandkumar, T. Y. Tsai, T. H. Kao, B. S. Inbaraj, and B. H. Chen, "Cytotoxicity and antibacterial activity of gold-supported cerium oxide nanoparticles," International Journal of Nanomedicine, vol. 9, pp. 5515-5531, 2014.

[67] L. Wang, S. Li, J. Yin et al., "The Density of Surface Coating Can Contribute to Different Antibacterial Activities of Gold Nanoparticles," Nano Letters, vol. 20, no. 7, pp. 5036-5042, 2020.

[68] S. Yougbare, T. K. Chang, S. H. Tan et al., "Antimicrobial Gold Nanoclusters: Recent Developments and Future 
Perspectives," International Journal of Molecular Sciences, vol. 20, no. 12, article 2924, 2019.

[69] S. A. Jalil, M. Akram, J. A. Bhat et al., "Creating superhydrophobic and antibacterial surfaces on gold by femtosecond laser pulses," Applied Surface Science, vol. 506, article 144952, 2020.

[70] M. Pišlová, K. Kolářová, B. Vokatá et al., “A new way to prepare gold nanoparticles by sputtering - Sterilization, stability and other properties," Materials Science \& Engineering, C: Materials for Biological Applications, vol. 115, article 111087, 2020.

[71] J. Simon, S. Udayan, E. S. Bindiya, S. G. Bhat, V. P. N. Nampoori, and M. Kailasnath, "Optical characterization and tunable antibacterial properties of gold nanoparticles with common proteins," Analytical Biochemistry, vol. 612, article 113975, 2021.

[72] I. M. Ahmady, M. K. Hameed, A. M. Almehdi et al., "Green and cytocompatible carboxyl modified gold-lysozyme nanoantibacterial for combating multidrug-resistant superbugs," Biomaterials Science, vol. 7, no. 12, pp. 5016-5026, 2019.

[73] M. Khosravi, A. Mirzaie, A. B. Kashtali, and H. Noorbazargan, "Antibacterial, anti-efflux, anti-biofilm, anti-slime (exopolysaccharide) production and urease inhibitory efficacies of novel synthesized gold nanoparticles coated Anthemis atropatana extract against multidrug- resistant Klebsiella pneumoniae strains," Archives of Microbiology, vol. 202, no. 8, pp. 2105-2115, 2020.

[74] S. Ranjan Sarker, S. A. Polash, J. Boath et al., "Functionalization of Elongated Tetrahexahedral Au Nanoparticles and Their Antimicrobial Activity Assay," ACS Applied Materials \& Interfaces, vol. 11, no. 14, pp. 13450-13459, 2019.

[75] J. Penders, M. Stolzoff, D. J. Hickey, M. Andersson, and T. J. Webster, "Shape-dependent antibacterial effects of noncytotoxic gold nanoparticles," International Journal of Nanomedicine, vol. 12, pp. 2457-2468, 2017.

[76] S. Hameed, Y. Wang, L. Zhao, L. Xie, and Y. Ying, "Shapedependent significant physical mutilation and antibacterial mechanisms of gold nanoparticles against foodborne bacterial pathogens (Escherichia coli, Pseudomonas aeruginosa and Staphylococcus aureus) at lower concentrations," Materials Science \& Engineering, C: Materials for Biological Applications, vol. 108, article 110338, 2020.

[77] W. C. Hu, M. R. Younis, Y. Zhou, C. Wang, and X. H. Xia, "In Situ Fabrication of Ultrasmall Gold Nanoparticles/2D MOFs Hybrid as Nanozyme for Antibacterial Therapy," Small, vol. 16, no. 23, article e2000553, 2020.

[78] F. Brasili, A. Capocefalo, D. Palmieri et al., "Assembling patchy plasmonic nanoparticles with aggregation-dependent antibacterial activity," Journal of Colloid and Interface Science, vol. 580, pp. 419-428, 2020.

[79] N. B. Arweiler and L. Netuschil, "The Oral Microbiota," Advances in Experimental Medicine and Biology, vol. 902, pp. 45-60, 2016.

[80] S. S. Oberoi, C. Dhingra, G. Sharma, and D. Sardana, "Antibiotics in dental practice: how justified are we," International Dental Journal, vol. 65, no. 1, pp. 4-10, 2015.

[81] W. Song and S. Ge, "Application of Antimicrobial Nanoparticles in Dentistry," Molecules, vol. 24, no. 6, article 1033, 2019.

[82] I. Venditti, "Nanostructured Materials Based on Noble Metals for Advanced Biological Applications," Nanomaterials, vol. 9, no. 11, article 1593, 2019.
[83] M. Zhang, X. Liu, Y. Xie et al., "Biological Safe Gold Nanoparticle-Modified Dental Aligner Prevents thePorphyromonas gingivalisBiofilm Formation," ACS Omega, vol. 5, no. 30, pp. 18685-18692, 2020.

[84] Y. Y. Wu, P. Huang, and F. Y. Wu, "A label-free colorimetric aptasensor based on controllable aggregation of AuNPs for the detection of multiplex antibiotics," Food Chemistry, vol. 304, article 125377, 2020.

[85] Z. Zhang, Y. Tian, P. Huang, and F. Y. Wu, "Using targetspecific aptamers to enhance the peroxidase-like activity of gold nanoclusters for colorimetric detection of tetracycline antibiotics," Talanta, vol. 208, article 120342, 2020.

[86] Y. Li, Q. Du, X. Zhang, and Y. Huang, "Ratiometric detection of tetracycline based on gold nanocluster enhanced $\mathrm{Eu}^{3+}$ fluorescence," Talanta, vol. 206, article 120202, 2020.

[87] Y. Leng, Y. Fu, Z. Lu et al., "Sub-10-nm multicolored gold nanoparticles for colorimetric determination of antibiotics via formation of interlocking rings," Mikrochimica Acta, vol. 186, no. 12, article 803, 2019.

[88] K. Jadhav, R. Hr, S. Deshpande et al., "Phytosynthesis of gold nanoparticles: characterization, biocompatibility, and evaluation of its osteoinductive potential for application in implant dentistry," Materials Science \& Engineering. C, Materials for Biological Applications, vol. 93, pp. 664-670, 2018.

[89] D. N. Heo, W. K. Ko, H. R. Lee et al., “Titanium dental implants surface-immobilized with gold nanoparticles as osteoinductive agents for rapid osseointegration," Journal of Colloid and Interface Science, vol. 469, pp. 129-137, 2016.

[90] Y. Xia, H. Chen, F. Zhang et al., "Gold nanoparticles in injectable calcium phosphate cement enhance osteogenic differentiation of human dental pulp stem cells," Nanomedicine, vol. 14, no. 1, pp. 35-45, 2018.

[91] J. Guo, K. Rahme, Y. He, L. L. Li, J. D. Holmes, and C. M. O'Driscoll, "Gold nanoparticles enlighten the future of cancer theranostics," International Journal of Nanomedicine, vol. 12, pp. 6131-6152, 2017.

[92] N. S. Aminabad, M. Farshbaf, and A. Akbarzadeh, "Recent Advances of Gold Nanoparticles in Biomedical Applications: State of the Art," Cell Biochemistry and Biophysics, vol. 77, no. 2, pp. 123-137, 2019.

[93] S. R. Satapathy, A. Nayak, S. Siddharth, S. Das, D. Nayak, and C. N. Kundu, "Metallic gold and bioactive quinacrine hybrid nanoparticles inhibit oral cancer stem cell and angiogenesis by deregulating inflammatory cytokines in p53 dependent manner," Nanomedicine, vol. 14, no. 3, pp. 883-896, 2018.

[94] P. Durgesh, S. Sridharan, S. K. Prabhu, R. Rao, V. Rudresh, and D. H. Bangalore, "Microbial contamination and plaque scores of nanogold-coated toothbrush," International Journal of Dental Hygiene, vol. 18, no. 3, pp. 278-284, 2020.

[95] C. Colombé, X. Le Guével, A. Martin-Serrano et al., "Gold nanoclusters as a contrast agent for image-guided surgery of head and neck tumors," Nanomedicine, vol. 20, article 102011, 2019.

[96] C. S. Kim, D. Ingato, P. Wilder-Smith, Z. Chen, and Y. J. Kwon, "Stimuli-disassembling gold nanoclusters for diagnosis of early stage oral cancer by optical coherence tomography," Nano Convergence, vol. 5, no. 1, p. 3, 2018.

[97] D. Chakraborty, T. S. Viveka, K. Arvind et al., "A facile gold nanoparticle-based ELISA system for detection of 
osteopontin in saliva: towards oral cancer diagnostics," Clinica Chimica Acta, vol. 477, pp. 166-172, 2018.

[98] C. Li, Z. Li, Y. Zhang, A. H. Fathy, and M. Zhou, "The role of the $\mathrm{Wnt} / \beta$-catenin signaling pathway in the proliferation of gold nanoparticle-treated human periodontal ligament stem cells," Stem Cell Research \& Therapy, vol. 9, no. 1, p. 214, 2018.

[99] U. Daood, Z. Akram, J. P. Matinlinna, and A. S. Fawzy, “Dentine collagen cross-linking using tiopronin-protected $\mathrm{Au} / \mathrm{EDC}$ nanoparticles formulations," Dental Materials, vol. 35, no. 7, pp. 1017-1030, 2019.

[100] J. Junevičius, J. Žilinskas, K. Česaitis, G. Česaitienė, D. Gleiznys, and Ž. Maželienè, "Antimicrobial activity of silver and gold in toothpastes: a comparative analysis," Stomatologija, vol. 17, no. 1, pp. 9-12, 2015.

[101] J. Zhu, S. Liu, T. Zhang et al., "Porous gold layer coated silver nanoplates with efficient antimicrobial activity," Colloids and Surfaces, B: Biointerfaces, vol. 186, article 110727, 2020.

[102] O. Chlumsky, S. Purkrtova, H. Michova Turonova et al., "The effect of gold and silver nanoparticles, chitosan and their combinations on bacterial biofilms of food-borne pathogens," Biofouling, vol. 36, no. 2, pp. 222-233, 2020.

[103] K. Saravanakumar, A. V. A. Mariadoss, A. Sathiyaseelan, and M. H. Wang, "Synthesis and characterization of nanochitosan capped gold nanoparticles with multifunctional bioactive properties," International Journal of Biological Macromolecules, vol. 165, pp. 747-757, 2020.

[104] P. Chandran, J. E. Riviere, and N. A. Monteiro-Riviere, "Surface chemistry of gold nanoparticles determines the biocorona composition impacting cellular uptake, toxicity and gene expression profiles in human endothelial cells," Nanotoxicology, vol. 11, no. 4, pp. 507-519, 2017.

[105] M. van Pomeren, W. J. G. M. Peijnenburg, R. C. Vlieg, S. J. T. van Noort, and M. G. Vijver, "The biodistribution and immuno-responses of differently shaped non-modified gold particles in zebrafish embryos," Nanotoxicology, vol. 13, no. 4, pp. 558-571, 2019.

[106] N. K. Hante, C. Medina, and M. J. Santos-Martinez, "Effect on Platelet Function of Metal-Based Nanoparticles Developed for Medical Applications," Frontiers in Cardiovascular Medicine, vol. 6, p. 139, 2019.

[107] S. Riaz, N. Fatima Rana, I. Hussain et al., "Effect of FlavonoidCoated Gold Nanoparticles on Bacterial Colonization in Mice Organs," Nanomaterials, vol. 10, no. 9, article E1769, p. 1769, 2020. 